\title{
The Ultimate Detection Limit: Building Electron Diffraction Patterns One Electron at a Time
}

Christian Kisielowski ${ }^{1}$, Petra Specht ${ }^{2}$, David Yancey ${ }^{3}$, Steve Rozeveld ${ }^{3}$, Joo Kang ${ }^{3}$, Alyssa McKenna ${ }^{3}$ and David Barton ${ }^{3}$

${ }^{1}$ The Molecular Foundry and Joint Center for Artificial Photosynthesis, Berkeley, California, United States, ${ }^{2}$ University of California in Berkeley, Berkeley, California, United States, ${ }^{3}$ Dow, Midland, Minnesota, United States

The wave/particle dualism of electrons is well documented by double-slit experiments [1] that demonstrate how quantum mechanical wave functions collapse and emerge in the macroscopic world that surrounds us. In electron microscopy, this subject gained renewed attention with the demonstration that a modulation of the sample illumination in the time domain greatly affects beam-sample interactions [2,3]. These findings raise questions about persistent misconceptions [4] and the entanglement and decoherence in electron microscopy [5].

Here we report on experiments that track the occurrence of individual scattering events in the quantum mechanical limit were single electrons interfere with themselves. We show in this ultimate detection limit how diffraction patterns are created one electron at a time instead of merely recording how diffraction intensities can decay with increasing dose accumulation if the investigated samples are radiation sensitive. For this purpose we take advantage of a Nelsonian (pencil-like) illumination scheme that allows restricting the area irradiated by an electron beam of outstanding coherence [2] and by employing the K2 camera in the TEAM I microscope operated at $300 \mathrm{kV}$. It is seen in Figures 1a and $1 \mathrm{~b}$ that this approach indeed captures single electron scattering events. Successively, a larger electron dose can be accumulated by recording and processing image series to build up the entire diffraction pattern of crystals (Figures 1c and $1 \mathrm{~d}$ ) in analogy to the detection of single electrons that were used in the past to build up the diffraction pattern of a double slit and reveal the wave/particle dualism of electrons [1]. A Double Metal Cyanide (DMC) catalyst $\left(\mathrm{M}_{\mathrm{x}}{ }^{1}\left[\mathrm{M}^{2}(\mathrm{CN})_{6}\right]_{\mathrm{y}}\left(\mathrm{M}^{1}=\mathrm{Zn} ; \mathrm{M}^{2}=\mathrm{Co} ; \mathrm{Fe}\right)\right.$, Figure 2 a $)$ [6] was chosen for these experiments because it looses structural integrity in the electron beam at an accumulated dose of a few electrons per square Ångstrom just as biological substances often do. Unexpectedly, it is seen in Figure $2 \mathrm{~b}$ that a dose accumulation does not yield to an instant loss of diffraction intensity in this case. Instead, a linear growth of the diffraction peaks is recorded even if scattering vectors are large. In this talk we discuss existing differences between the traditional recording of electron diffraction patterns and their formation in the ultimate detection limit [7]. 

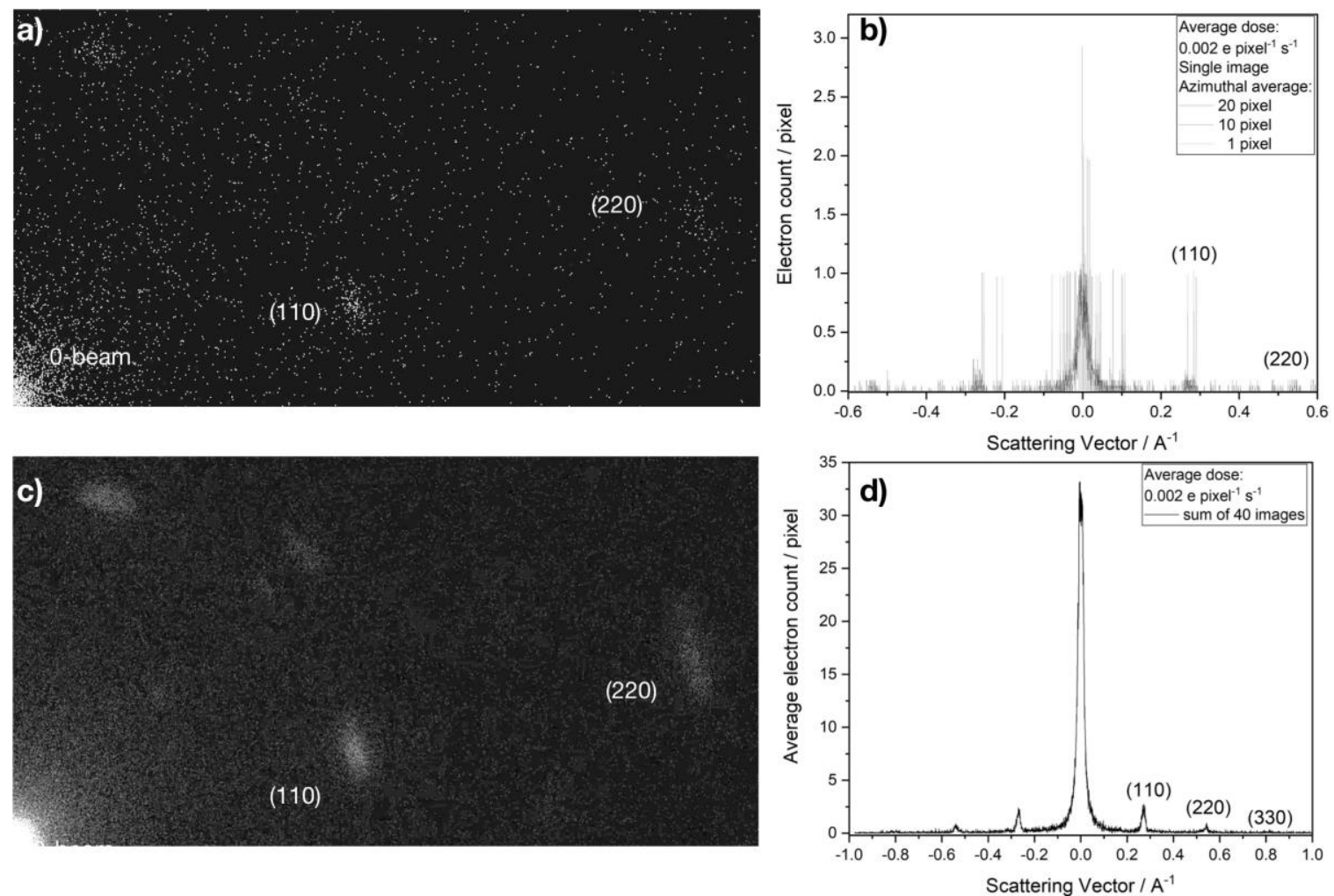

Figure 1. Building up the diffraction pattern of DMC one electron at a time. The irradiated sample area is $106 \AA 2$. a) Single image, dose rate 0.01 e $\AA-2 s-1$. White dots capture the detection of a single electron. b) Line profiles across diffraction spots for 3 different values of azimuthal average. The average electron count drops well below 1 electron / pixel. c) Average of 40 accumulated images, total dose: 0.4 e A-2. d) Line profile across the averaged image.
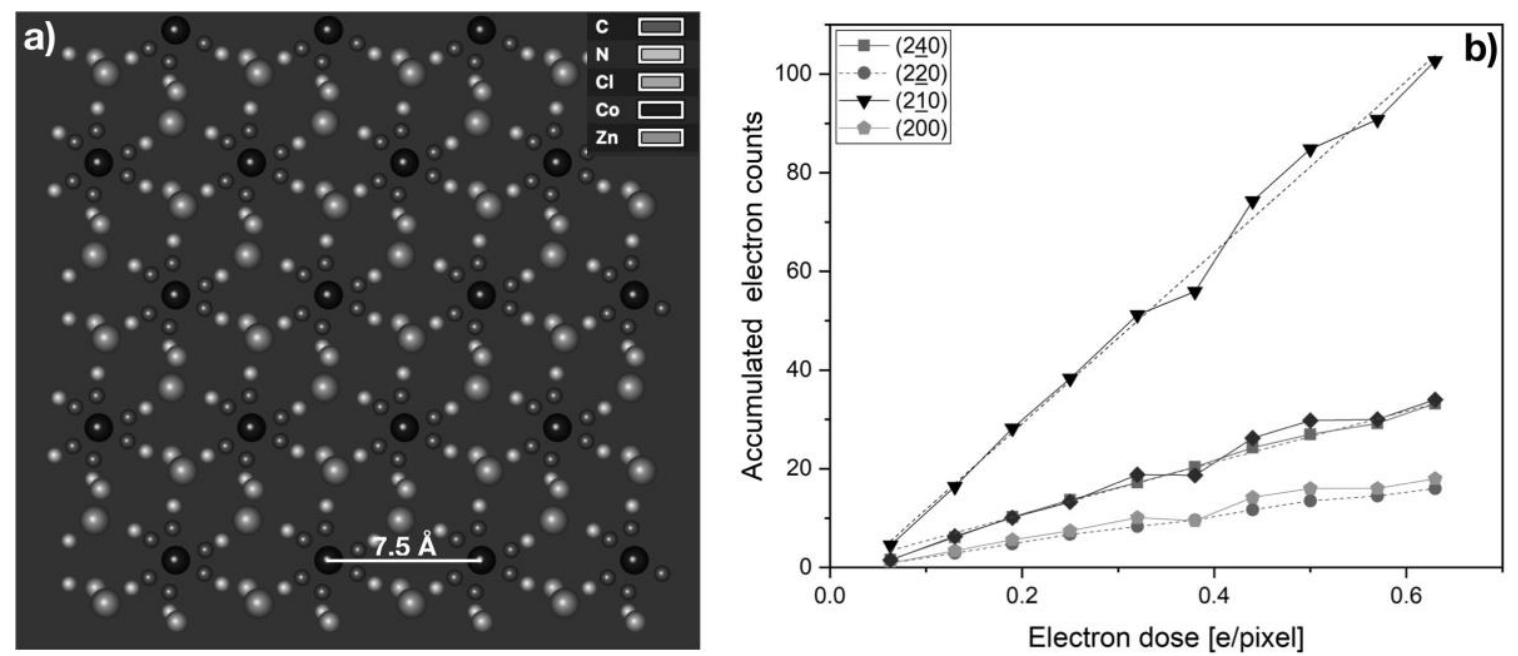

Figure 2. a) Crystal structure of DMC [6]. b) Linear growth of diffraction spots with dose accumulation including large scattering vectors e.g. $g(240)=1 / 1.8 \AA-1$.

References

[1] C Jönsson 1961 Zeitschrift für Physik 161 454-474; P G Merli, G F Missiroli, G Pozzi 1976 
American Journal of Physics 44 306-7; H. Lichte, https://gigamove.rz.rwthaachen.de/d/id/JDfcpin7bJbyde; A Tonomura, J Endo, T Matsuda, T Kawasaki and H Ezawa 1989, American Journal of Physics 57 117-120

[2] C. Kisielowski, Specht P., Freitag B., Kieft E.R., Verhoeven W., van Rens J.F.M., Mutsaers P., Luiten J., Rozeveld, S., Kang J., McKenna A.J., Nickias P., Yancey D.F., Adv. Func. Mater. 29, 1807818, 2019 [3] VandenBussche, E. J.; Flannigan, D. J., Nano Lett. 19, 6687-6694, 2019

[4] D. van Dyck, Ultramicroscopy 111, 894-900, 2011

[5] P. Schattschneider, S. Löffler, Ultramicroscopy 190, 39-44, 2018

[6] J. C. Wojdeł, S.T. Bromley, F. Illas, J. C. Jansen, J. Mol. Model. 13, 751-756, 2007

[7] The work at the Molecular Foundry was supported by the DOE Office of Science, Office of Basic Energy Sciences, under contract DE-AC02-05-CH11231 and a CRADA. 